\title{
EL PRINCIPIO DE IGUALDAD Y NO DISCRIMINACIÓN ANALIZADO DESDE LA FIGURA DE LA MUJER COMO SUJETO DE DERECHOS.
}

\author{
Abg. Andrea Gabriela Zuleta Sánchez, Mgs. \\ Magíster en Derecho Constitucional (Ecuador). \\ Docente Tiempo Completo de la Facultad de Derecho y Gobernabilidad \\ de la Universidad Tecnológica ECOTEC, Ecuador. \\ azuleta@ecotec.edu.ec
}

\section{ARTÍCULO DE REFLEXIÓN}

Recibido: 12 de julio de 2019.

Aceptado: 9 de septiembre de 2019.

\section{RESUMEN}

El presente artículo, tiene como objetivo general analizar a través de un estudio doctrinario y crítico, el principio de igualdad tanto formal como material, el principio de igualdad y no discriminación analizado desde la figura de la mujer como sujeto de derechos. El bloque de constitucionalidad, es decir, la Constitución junto con los instrumentos internacionales de derechos humanos ratificados por el Ecuador garantiza la igualdad como piedra angular del Estado constitucional de derechos. A través de la subsunción entre la norma y el hecho jurídico, se identifican políticas estatales dirigidas a la implementación real del principio de igualdad desde el enfoque de género, mediante un estudio doctrinario y normativo del derecho de los derechos humanos. Por lo que se concluye que el principio de igualdad no obstante se encuentra contenido en normativa tanto nacional como internacional debe ser ejercido por cada individuo, como titular de derechos, y que el Estado, como portador de obligaciones, es quien debe garantizar su aplicación efectiva en todos los ámbitos. La metodología empleada ha sido el uso del método deductivo y la investigación jurídica mixta (investigación teórica y empírica), utilizando además el derecho comparado.

Palabras Clave: igualdad material y formal, derechos de las mujeres, derechos humanos, estado constitucional de derechos.

\section{ABSTRACT}

The general objective of this article is to analyze, through a doctrinal and critical study, the principle of equality, both formal and material, the principle of equality and non-discrimination analyzed from the role of women as a subject of rights. The constitutionality group, that is, the Constitution with the international 
human rights instruments ratified by Ecuador, guarantees equality as the cornerstone of the Constitutional State of rights. Throughout the subsumption between the law and the legal fact, state policies are identified aimed at the real implementation of the principle of equality from the gender approach. It is done through a doctrinal and normative study of human rights law. So it is concluded that the principle of equality is contained in both national and international regulations must be exercised by each individual, as the bearer of rights, and that the State, as bearer of obligations, is the one who must ensure its effective application in all fields. Dedductive methodology has been used and mixed legal research (theoretical and empirical research), using comparative law as well.

Keywords: material and formal equality, women's rights, human rights, constitutional status of rights.

\section{INTRODUCCIÓN}

Igualdad, libertad y justicia, son parte de los principios inspiradores del derecho moderno, que devienen en las diferentes luchas sociales ocurridas en el mundo contemporáneo, en aras de conseguir derechos que otrora no existían. La igualdad sin duda alguna, cimienta el estado constitucional de derechos, ya que dicho principio, equilibra la balanza para todos los actores de la sociedad. Ya que como tal, configura un escenario donde todos los ciudadanos son iguales ante la ley. Los seres humanos son distintos unos de otros, en fondo y en forma, pero dicha diferencia, no necesariamente significa que deba existir desequilibrio. Thomas Hobbes, en su Leviatán manifiesta que el problema de los seres humanos, es su igualdad en fuerza y en inteligencia generalmente, lo cual deviene en condiciones de violencia intrínseca o en "el estado de naturaleza" (Hobbes, 2010). La igualdad, en toda la dimensión del término, se constituye en una quimera, pero esa debería ser la motivación principal, para que el Estado, vaya más allá, y continúe implementando medidas que permitan la consolidación de la perfectibilidad de la igualdad, principalmente en el goce de los derechos, por parte de los grupos que se encuentran en el lugar menos ventajoso de la balanza, como en este caso, la mujer.

En tal sentido, nace el debate entre igualdad formal e igualdad material, que según Ricardo Ruiz Carbonell, el primero comprende "las expresiones intrínsecas del principio de igualdad de trato; es decir, en el contenido de la ley y a su vez el mandato de no discriminación" y "el mandato para los poderes públicos de remover los obstáculos a la igualdad de los hechos, lo que puede llegar a suponer e incluso a exigir, la implantación de algunas medidas que compensen diversas diferencias negativas, encontrando su punto de partida en la formalización específica jurídica frente y contra las discriminaciones en sentido estricto" (Ruiz, s.f.). 
Las mujeres, en vista de dicha desigualdad encarnada en una sociedad tradicionalmente machista, a lo largo de los años ha sido testigo de cómo sus derechos han debido ser reivindicados, por la lucha permanente en la que se han enfocado y en la que continúan por los diferentes desfases que siguen afectando a su población. Uno de estos desfases es la violencia contra la mujer por el hecho de ser mujer. En el Ecuador 6 de cada 10 mujeres han sido víctimas de algún tipo de violencia (INEC, 2012).

La importancia del abordaje de este tema radica en la identificación de las situaciones de desigualdades entre hombres y mujeres debido a los estereotipos, construcciones sociales y culturales desfiguradas que nominan lo masculino y lo femenino asignándoles características inequitativas y excluyentes entre sí. Así también, es necesario abordar parte de la historia común del pueblo latinoamericano, que sitúa a la violencia en contra de mujeres y niñas, como parte de una oprobiosa costumbre arraigada a través de los tiempos. Es notorio desde esa perspectiva que el objeto de este recorrido no solo implica un estudio legal sino también al tejido diverso que conforma la construcción de género. Finalmente, esta investigación tiene como objetivo general analizar el principio de Igualdad, respecto a las relaciones entre hombres y mujeres que se obtiene a través de la revisión tanto de normativa nacional como internacional y de la doctrina pertinente.

\section{Principio de Igualdad}

Encontrándose en un escenario, que ha mutado desde sus transformaciones, y se mantiene en un proceso de construcción continua, a decir de Carbonell, uno de los primeros pasos que se debe dar como sociedad, es el reconocimiento de los derechos humanos, porque sin ellos la vida humana carecería de significado y sus más altos valores como la igualdad, la libertad y la justicia, serían poco menos que promesas vanas e irrealizables. Sin una cultura que priorice los derechos será imposible cambiar una realidad que nos ofende y lastima profundamente (Carbonell, 2001).

La Constitución del Ecuador del 2008 que en adelante se nombra CRE, en su preámbulo contiene los fundamentos sobre los que se asienta el Estado ecuatoriano, encontrándose en estos, el reconocimiento de la calidad de herederos de diferentes luchas sociales frente a toda forma de dominación y colonización (Asamblea Constituyente, 2008). Este fundamento ha sido una constante, en la realidad ecuatoriana, ya que con el paso del tiempo ha cambiado el objetivo, más no la lucha reivindicatoria en sí. En ese contexto, es necesario sumergirse en el principio de igualdad, que constituye el principio fundamental de las relaciones personales de todo tipo, en un Estado constitucional de derechos, es por ello que la CRE, en 
su artículo 11, proclama el ejercicio pleno de los derechos constitucionales, sin restricción o condición alguna, manifestando la igualdad general de las personas, dotándolas de derechos, deberes y oportunidades sin restricciones, agregando además una prohibición de discriminación en cualquiera de sus formas; es decir, sus creencias, sus orígenes, su orientación social política sean estas distinciones, personales o colectivas, temporales o permanentes y que dicha distinción busque generar un menoscabo o anular el reconocimiento, goce o ejercicio de los derechos. Para lo que la ley sancionará, a cualquier manifestación o forma de discriminación, generando además una obligación estatal de adoptar medidas de acción afirmativa que tengan como finalidad promover una igualdad real a favor de quienes se encuentren en situación de desigualdad como titulares de derechos (Asamblea Constituyente, 2008).

La CRE se encuentra orientada hacia la búsqueda de la igualdad como principio, igualdad que va más allá de la declaración normativa. Para ello resulta imperante, continuar con la ruptura de los paradigmas en los que se cimienta la sociedad tradicional. Así, para la tratadista Alda Facio, significa hacer de esta disciplina un instrumento transformador que desplace los actuales modelos sexuales, sociales, económicos y políticos hacia una convivencia humana basada en la aceptación de la otra persona como una legítima otra y en la colaboración como resultante de dicho respeto a la diversidad (Facio, 1999).

Ahora bien, el acceso a los derechos, deberes y oportunidades garantizados por la CRE, es fácticamente posible, siempre y cuando el Estado adopte mecanismos y políticas públicas que garanticen dicha igualdad, sobre todo en consideración de los grupos de atención prioritaria y en situación de vulnerabilidad. En dicho espectro se refiere al género, el cual, en una sociedad ideal no debería ser una causa para diferenciar en la garantía de derechos. No obstante, la sociedad contemporánea en el país se cimienta en un enfoque patriarcal que se sostiene sobre la normalización del machismo y una preferencia ampliamente asumida hacia la masculinidad.

El patriarcado, es la manifestación social en la cual se instituye al hombre, sinónimo de masculino, como señor de la sociedad, en virtud de sus cualidades implícitas al hecho de ser hombre. Este escenario conlleva a una degradación de la figura femenina, desde la sima de las relaciones sociales puesto que, desde el nacimiento, se orienta a una situación de poder entre los niños y las niñas, lo que degenera en el machismo y otros problemas sociales. Según Gerda Lerner implica que los varones tienen poder en todas las instituciones importantes en la sociedad y que se priva a las mujeres del acceso de las mismas pero no implica que las mujeres no tengan, ningún tipo de poder ni derechos, influencias o recursos (Lerner, 1986). 
La CRE, al inspirarse en principios como la equidad, la libertad, la vida, la dignidad humana, principios que se van desarrollando a través de todo el cuerpo normativo, además reconoce la protección de los grupos sociales que históricamente se han encontrado en desventajas, ya que se han visto diferenciados, relegados y en ocasiones discriminados, por los grupos "privilegiados" o parte de las grandes mayorías, buscando de esta forma equilibrar la balanza a fin de garantizar el pleno goce de los derechos. Así la CRE, en su artículo 66 numeral 4, reconoce y garantiza, el derecho a la igualdad, dentro de la cual se encuentra además de la igualdad formal, material el principio a la no discriminación, que constituye un paso importante concordante con el Estado constitucional de derechos (Asamblea Constituyente, 2008).

Además, a decir de la jurista Andrea Cajas Córdova, quien contempla al derecho a la igualdad, la Constitución ecuatoriana vigente, logra trascender más allá de lo estrictamente formal lo que, aunque se interpreta como progresión en derechos, debe reconocerse que resulta insuficiente la sola declaratoria para alcanzar una efectiva realización. Por ello, la Constitución reconoce y garantiza los tres principios, es decir igualdad tanto formal como material y no discriminación, mismos que se encuentran considerados como la base angular de la igualdad de género (Cajas, 2011).

\section{Igualdad formal}

El principio de igualdad tal como se indica en la Declaración Universal de Derechos Humanos en su primer artículo expresa que los seres humanos, nacen libres e iguales, y adiciona que esta igualdad aplica en dignidad de derechos. Además, en el artículo segundo manifiesta que toda persona, sin realizar ningún tipo de distinciones, tiene los derechos y libertades proclamados en dicha declaración, indistintamente de ninguna diferencia de cualquier índole que pudiera existir. De lo que se colige que la igualdad es inherente a la dignidad humana, y en base a esto quedaría prohibida cualquier vulneración que limite el ejercicio y goce de los derechos atribuidos al ser humano en virtud de su dignidad.

El principio de igualdad, además está recogido en el Pacto Internacional de Derechos Civiles y Políticos y en el Pacto de Derechos Económicos Sociales y Culturales ambos suscritos en el año 1966, que fueron los dos primeros tratados internacionales vinculantes que se desplegaron en virtud de la Declaración Universal de Derechos Humanos. De igual forma, ocurre con otros instrumentos internacionales de derechos humanos, tanto en el Sistema Universal de la Organización de las Naciones Unidas (ONU) como en el Sistema Regional de Protección de Derechos de la Organización de Estados Americanos (OEA). 
El Comité de Derechos Económicos Sociales y Culturales, ha señalado que las garantías de no discriminación e igualdad en los instrumentos internacionales de Derechos Humanos prevén la igualdad tanto de facto como de jure (Comité de Derechos Económicos Sociales y Culturales, 2005). Categorías que, no obstante, son conceptos diferentes; pero se interrelacionan reciprocamente. Así, la igualdad formal debe cristalizarse en la igualdad material, sino carecería de utilidad el mantener una norma que implique la igualdad ante la ley si esta no se hace real o práctica.

Por ello, se puede inferir que en cuanto al último inciso del artículo 11, numeral 2 de la Constitución, que expresa como obligación estatal la adopción de medidas de acción afirmativa, que promuevan la igualdad real, señalando que la misma será en favor de los titulares de derechos que se encuentren en situación de desigualdad, comprende que todos somos diferentes, pero tenemos derecho a las mismas oportunidades, según la especificidad de nuestro ser. Esta situación, según Daniel Muñoz, encuentra ciertas limitaciones, en tanto que "políticamente es imposible conseguir los objetivos que se marca nuestra Constitución. Se ha de entender más como una conquista en aras de una democracia efectiva, que como mantener que todos tengamos un pleno desarrollo social como individuos o grupos" (Muñoz Cabrera, 2010, p. 406).

La igualdad de jure o igualdad formal, según el Tratadista Luigi Ferrajioli: "es la relación que unifica a una clase de sujetos en la titularidad de aquellos derechos -en cuanto reconocidos y garantizados a todos y en igual medida- son llamados universales o fundamentales" (Ferrajioli, 2010, p. 166). Así, la igualdad formal consiste en la igualdad ante la ley. Este principio garantiza la igualdad de trato a todos los destinatarios de la norma jurídica y la ausencia de todo privilegio en materia de jurisdicción e impuestos (Carmona, 1994).

Con la finalidad de adecuar el principio de igualdad a la realidad jurídica de la sociedad, el Estado debe implementar las políticas recomendadas por el Comité de Derechos Económicos, Sociales y Culturales, el cual observa que los estados partes del Pacto Internacional de Derechos Económicos, Sociales y Culturales tienen la obligación de respetar, proteger y de cumplir con dicho principio. En aras de respetar la igualdad, en este caso formal, el Comité expresa que los Estados deberán abstenerse de realizar actos discriminatorios que directa o indirectamente tengan como resultado la omisión del reconocimiento de la igualdad de derechos del hombre y la mujer, al disfrute de los derechos económicos, sociales y culturales. Los Estados no aprobarán o derogarán las leyes y deberán rescindir las políticas, las disposiciones administrativas y los programas que no están conformes con el derecho protegido en el artículo 3 , que 
manifiesta el compromiso de "asegurar a los hombres y a las mujeres igual título a gozar de todos los derechos económicos, sociales y culturales".

En particular, los estados partes tomarán en cuenta la manera en que la aplicación de normas y principios jurídicos aparentemente neutrales en lo que se refiere al género, tenga un efecto negativo en la capacidad del hombre y la mujer para disfrutar de sus derechos humanos en pie de igualdad y deberán reivindicar al afectado en el caso de que se estuviere limitando el goce de la igualdad. En cuanto a la obligación de proteger, exige que los estados partes, tomen disposiciones encaminadas directamente a la eliminación de los prejuicios, las costumbres y todas las demás prácticas que perpetúan la noción de inferioridad o superioridad de uno u otro sexo y las funciones estereotipadas del hombre y la mujer. La obligación de los estados partes de proteger el derecho enunciado en el artículo 3 del Pacto comprende, entre otros puntos, el respeto y la aprobación de disposiciones constitucionales y legislativas sobre la igualdad de derechos del hombre y la mujer a disfrutar de todos los derechos humanos y la prohibición de toda clase de discriminación, la aprobación de instrumentos legislativos que eliminen la discriminación e impidan a terceros perturbar directa o indirectamente el disfrute de este derecho, la adopción de medidas administrativas y programas, así como el establecimiento de instituciones públicas, organismos y programas para proteger a la mujer contra la discriminación.

La igualdad formal, es el principio por el cual el Estado de derechos, delimita la convivencia de la sociedad a través del parámetro de la norma cuyo origen deviene de la evolución de la misma sociedad a través del tiempo. La igualdad formal presupone que cada individuo del Estado, hombre o mujer, será tratado ante la ley de una forma que garantice el goce y ejercicio de sus derechos constitucionales; por ello, dicha incondicionalidad de la ley debe ser garantizada en todo aspecto en el que se aplique la norma.

EI CEDAW, (Comité de la Convención sobre la Eliminación de todas las formas de discriminación contra la Mujer), en la Recomendación General No. 25 consideró que el enfoque puramente formal, no es suficiente para lograr la igualdad de facto (igualdad material). Ya que es necesario que la mujer tenga las mismas oportunidades desde un primer momento y que cuente con un entorno que le lleve a obtener resultados iguales.

Es decir que el sistema como tal no este diseñado para limitar a la misma por lo que no alcanza con garantizar un trato idéntico al del hombre se requiere además una estrategia eficaz orientada a corregir la representación ineficiente de la mujer y una redistribución de los recursos y el poder entre el hombre y la mujer (Comite para la Eliminación de la Discriminación contra la Mujer, 1999). 
Evidentemente la brecha discriminatoria existente entre hombre y mujeres no solo es claramente marcada, sino además se perpetua en el tiempo, podría creerse que con la tecnología y el avance social disminuiría la brecha pero desgraciadamente tal como lo refiere el Informe de las Naciones Unidas, "ningún país del mundo, trata a sus mujeres tan bien como a sus hombres" (Desarrollo, 1995).

Especialmente en temas como el acceso a la educación, salud sexual y reproductiva, la igualdad de oportunidades en el trabajo, seguridad e integridad y la infinita gama de situaciones sociales en las que la mujer tiene derechos; pero no tan efectivos en comparación a los hombres (Chamberlain, 2003).

Basta con ver las noticias para inferir que el riesgo que viven las mujeres diariamente va en aumento en América Latina. Amenazas, violencia física, verbal y sexual, desapariciones forzosas, mutilaciones, femicidio se han convertido en una realidad diaria, que exige a la sociedad realizar una introspección, evidentemente tal como Katherine Bartlett sostiene, las reglas de la igualdad formal, no producen los mismos efectos por cuanto las significativas diferencias entre las características y circunstancias de las mujeres y los hombres (Bartlett, 1993).

\section{Igualdad material o sustantiva}

Inicialmente se abordó la igualdad formal y se reveló que esta se encuentra estrictamente ligada a la igualdad material, tanto que se encuentran enlazadas entre sí y una sin la otra no podría cumplir con su objetivo eficientemente. La igualdad de hecho en conjunto a la igualdad formal, conforman los pilares de la igualdad de género. La igualdad sustancial, material o de hecho, presupone, según Pietro Sanchís, "una igualdad real y efectiva, que llama a superar la escueta igualdad jurídica tradicional, ya que exige la intervención estatal y de la población, en el plano económico y social, para eliminar o remediar los escenarios de desigualdad" (Pietro Sanchís, 1994).

La igualdad sustancial, implica el reconocimiento de un derecho formal, es decir, tiene naturaleza prestacional en tanto se contrapone el ordenamiento y su imperativo categórico contenido para con la realidad y el cumplimiento de la obligación contenida en dicho imperativo. De ello se desprenden tres aristas. La primera es cuando la igualdad material viene apoyada por un derecho fundamental de naturaleza prestacional directamente exigible. La segunda tiene lugar el presupuesto de que una pretensión de igualdad sustancial concurre con otro derecho fundamental, aun cuando no sea de naturaleza prestacional. A decir de Pietro Sanchís, se trata de una cierta presunción de que el bien jurídico 
tutelado es valioso y merece protección, ya que de los múltiples objetivos que pueden perseguir las acciones de discriminación inversa, hay algunos que aparecen privilegiados en la Constitución, representando en consecuencia una razón fuerte en favor de la adopción de medidas de igualación material (Pietro, 1994). Y el tercer presupuesto, es cuando una pretensión de igualdad material viene acompañada por una exigencia de igualdad formal.

El Comité de la CEDAW, en la precitada Recomendación General No. 25, expresa que un enfoque jurídico o programático puramente formal no es suficiente para lograr la igualdad de facto con el hombre, que el Comité interpreta como igualdad sustantiva. Además, la Convención requiere que la mujer tenga las mismas oportunidades desde un primer momento y que disponga de un entorno que le permita conseguir la igualdad de resultados. El logro del objetivo de la igualdad sustantiva también exige una estrategia eficaz encaminada a corregir la representación insuficiente de la mujer y una redistribución de los recursos y el poder entre el hombre y la mujer (Comité para la Eliminación de la Discriminación contra la Mujer, 1999).

Así, la igualdad material es la realización de la igualdad formal, puesto que la sociedad se encuentra sobrepuesta en un andamiaje imperfecto el cual está gobernado por la ley cuya consecuencia es la igualdad material, siendo el Estado el obligado a exigir el cumplimiento de la misma. El principio de igualdad material o real suele entenderse como una reinterpretación del principio de igualdad formal en el Estado Social de Derecho (Carmona, 1994).

El principio de igualdad material acuña varias teorías que refleja la cantidad de fuentes y los tipos de diferencia que existen, así como las alternativas que se puede implementar para contrarrestar estas diferencias. Citando a Ximena Avilés:

La igualdad sustantiva trata de remediar los efectos discriminatorios producidos en el pasado. Por ejemplo, como solamente las mujeres se embarazan, la aplicación del principio de igualdad formal implica una desventaja competitiva para ellas. La igualdad sustantiva procura neutralizar estas desventajas laborales a través del mejoramiento de los resultados: la creación de guarderías, el derecho de ausencia mesurado y compartido con el padre, entre otros (Avilés, 2017).

\section{No discriminación}

La no discriminación es consecuencia del principio de igualdad. El mismo radica en la reivindicación de los grupos de atención prioritaria y en situación de vulnerabilidad que han sido objeto de múltiples 
injusticias a lo largo de la historia. De esta idea se desprende que existen "categorías de personas" a las cuales el Estado debe garantizar el principio de igualdad prioritariamente con respecto de otras, no constituyendo esta misma una disonancia en relación al principio de igualdad. En palabras de Bounaventura de Souza Santos "tenemos el derecho a ser iguales cuando las diferencias nos interiorizan y a ser distintos cuando la igualdad nos descaracteriza" (De Souza, 2001), a través de la no discriminación se garantiza el efectivo goce de los derechos constitucionales por parte de la diversidad de los individuos en todas sus aristas, desde la raza, religión o etnia; así como por el género, orientación sexual o política.

Los grupos de personas denominadas usualmente como categorías sospechosas por parte de la doctrina, se encuentran establecidos como tal, en tanto que en el espacio temporal “...han sido objeto de prácticas discriminatorias en el pasado y como resultado persisten desigualdades de hecho y prejuicios enraizados que no permiten a sus integrantes desarrollar plenamente su personalidad en los distintos sectores de la vida social y política" (Nino, 2010).

Las categorías sospechosas son criterios utilizados tanto por el Estado como por los particulares con miras a realizar diferencias que nunca parecerían justificarse.

En términos de Cornella Ferreres:

(...) si una Constitución prohíbe la discriminación por razón de raza y de sexo, por ejemplo, es claro que se den dos cosas al mismo tiempo: por un lado, existe suficiente consenso en la comunidad en el sentido de que las desigualdades que vivieron en el pasado un grupo de personas por motivo de su raza y las que sufrieron las mujeres constituyen una discriminación injustificable; por el otro, que es de conocimiento que perviven los efectos de ese pasado discriminatorio y en consecuencia, es necesario dotar de especial protección a los miembros de esos grupos (Ferreres, 2007).

La CEDAW, en su artículo 1 define que la discriminación contra la mujer "denotará toda distinción, exclusión o restricción basada en el sexo que tenga por objeto o por resultado menoscabar o anular el reconocimiento, goce o ejercicio por la mujer, independientemente de su estado civil, sobre la base de la igualdad del hombre y la mujer, de los derechos humanos y las libertades fundamentales en las esferas política, económica, social, cultural y civil o en cualquier otra esfera" (Convencion Sobre la Eliminacion de todas las Formas de Discriminacion Contra la Mujer, 1979).

\section{CONCLUSIONES}


El principio de igualdad, es trascendental para la consecución de los objetivos de un Estado de Derechos, sobretodo el de garantizar igualdad a todos ciudadanos, especialmente de los grupos llamados vulnerables, incluyéndose dentro de ese grupo a la mujer, por las razones ya explicadas en el presente artículo. Este Principio es parte de las garantías que una persona sin importar su edad, género, preferencia sexual o política, debe gozar sin limitación alguna y no debe entenderse como una "prerrogativa especial" entregada por el Estado sino como un fin en sí mismo para conseguir, la tan anhelada igualdad sustancial, la cual no debería ser negada a ninguna persona.

Una sociedad equitativa es una sociedad en la que el acceso a los derechos está garantizado a cada uno de los ciudadanos, no obstante la desigualdad existente, en las sociedades mundiales, localmente en América Latina (producto del mestizasgo específicamente en el caso del Ecuador) se observan marcadas diferencias que denotan inequidad, en múltiples aspectos, entre ellos económicos, sociales, culturales y lógicamente, producto además de costumbres, patriarcales y de segregación social, inequidad producto del Género, producto al "papel" que asigna la sociedad a los grupos, especialmente a la Mujer, lo que conlleva en muchos casos especialmente en las regiones más apartadas de las grandes ciudades, no exista verdadera igualdad, se presenten variados tipos de violencia en contra de mujeres y niñas, sino que además se perpetúe, esa desigualdad, al negarles acceso a educación, lo que mantiene la desigualdad existente. Una sociedad equitativa, garantiza equilibrio familiar, social, cultural y económico, lo que se traduce en individuos que aportarán al Estado siendo sujetos activos de transformaciones sociales, eliminando la necesidad de compensaciones como el Bono de Desarrollo Humano por lo que no tendrían que convertirse en carga para el Estado.

Ciertamente, existen grandes avances, en el tema de la desigualdad, el hecho de que se hable de igualdad formal y material, de violencia de género, de inequidad, ya es un triunfo en sí mismo, existe aún un camino largo por transitar, pero es necesario para el efectivo goce de los derechos humanos, transitar aún más, como manifestaron los comités de la CEDAW y de la Convención de Belem do Pará; solo protegiendo y haciendo cumplir las normas, se podrá lograr la igualdad de la mujer y el hombre, si se mantiene el ritmo del progreso en materias de igualdad, ciertamente, se logrará equilibrar no solo la abalanza económica y social sino que se podrá disminuir categóricamente el índice de casos de Violencia, contra la mujer, como resultado de discriminación.

El trabajo de la mujer como víctima de desigualdad, debe ser la lucha por la reivindicación de los derechos, es evidente que la tecnología, ha sido un gran aliado, en la lucha contra todo tipo de desigualdad, ya que 
la presión social ejercida a través de las redes sociales, permite que de manera inmediata, se conozcan múltiples formas de discriminación, generando que el descontento o protesta social, asegure que el Estado continúe con la implementación de programas, medidas y formas que erradiquen la discriminación y la violencia, generando varias campañas tanto nacionales como locales que pretenden empoderar a las mujeres, con campañas que van desde la educación, el acceso a servicios de toda índole, el acompañamiento judicial, asegurando el acceso gratuito a la justicia, pasan hasta los medios de comunicación se influya a favor de la igualdad entre hombres y mujeres. Con acierto se puede resumir, que la igualdad formal se hace real o sus efectos son reales, en el momento de trascender a la igualdad material.

Al iniciar la redacción de este artículo se planteó como objetivo general analizar a través de un estudio doctrinario y crítico, el principio de igualdad tanto formal como material, el principio de igualdad y no discriminación analizado desde la figura de la mujer como sujeto de derechos, de la revisión de todos los trabajos bibliográficos, de múltiples índoles, utilizados en esta investigación se ha concluido que a pesar de la normativa existente tanto nacional como internacionalmente, no se puede hablar aun de una verdadera igualdad entre hombres y mujeres producto de convencionalismos sociales que dotan de características a los géneros. Se considera imperante, proponer no solo un seguimiento de la fenomenología observada y contrastada en el presente papel, analizar el principio de lgualdad desde otras aristas que permitan observar otros grupos los cuales también han sido invisibilizados históricamente.

\section{BIBLIOGRAFÍA}

Asamblea Constituyente. (2008). Asamblea Nacional. Recueprado de http://www.asambleanacional.gov.ec/documentos/constitucion_de_bolsillo.pdf

Avilés, X. (23 de 11 de 2017). Recuperado de http://www.flacso.org.ec/docs/safisaviles.pdf

Bartlett, T. K. (1993). Gender and Law. Boston: Little Brown and company.

Cajas, A. (Julio de 2011). Universidad Andina Simon Bolivar. Recuperado de http://repositorio.uasb.edu.ec/bitstream/10644/2804/1/RAA29\%20Andrea\%20Karolina\%20Cajas\%20C\%C3\%B3rdova\%2C\%20Igualdad\%20de\%20G\%C3\% A9nero\%20\%20la.pdf

Carbonell, M. (2001). Los derechos humanos en la actualidad: una visión desde México. México: Alfaomega. 
Carmona, E. (1994). El principio de igualdad material en la Jurisprudencia del Tribunal Constitucional. Revista de Estudios Politicos Nueva Epoca, 266.

Chamberlain, C. (2003). La Convención CEDAW conociendo derechos de la Mujer, un primer paso para su defensa. Universidad Complutense de Madrid, 37.

Comité de Derechos Económicos Sociales y Culturales. (2005). Observación general № 16: La igualdad de derechos del hombre y la mujer al disfrute de los derechos económicos, sociales y culturales (artículo 3 del Pacto Internacional de Derechos Económicos, Sociales y Culturales). Ginebra: Consejo Economico de las Naciones Unidas.

Comité para la Eliminación de la Discriminación contra la Mujer. (1999). Recomendación General No. 25 sobre el párrafo 1 del artículo 4 de la Convención para la eliminación de todas las formas de discriminación contra la mujer, referentes a medidas especiales de caracter especial.

Convencion Sobre la Eliminacion de todas las Formas de Discriminacion Contra la Mujer. (1979). Recuperado de ONU: http://www.un.org/womenwatch/daw/cedaw/text/sconvention.htm

De Souza, B. (13 de marzo de 2001). La igualdad no es suficiente. (Masiosare, Entrevistador)

Desarrollo, P. d. (1995). Reporte de Desarrollo Humano. Genero y Desarrollo. Ginebra: ONU.

Facio, A. (1999). Feminismo, Genero y Patriarcado. Santiago: Facio y Fries.

Ferrajioli, L. (2010). Igualdad y Diferencia. En M. d. Humanos, Igualdad y No Discriminación. El reto de la diversidad. Quito: V\&M Graficas.

Ferreres, V. (2007). Justicia Constitucional y Democracia. Madrid: Centro de Estudios Politicos y Constitucionales.

Hobbes, T. (18 de abril de 2010). Leviatán de Thomas Hobbes. Recuperado de http://www.dialogica.com.ar/unr/epicom/2009/10/hobbes-vs-rousseau.html

INEC. (28 de junio de 2012). INEC. Recuperado de http://www.ecuadorencifras.gob.ec/documentos/webinec/Infografias/violenciagenero.pdf

Lerner, G. (1986). The Creation of Patriarchy. Oxford University Press, 346.

Muñoz, D. (2010). Igualdad Jurídica o igualdad material, ¿que va antes el huevo o la gallina? Anuario de Derechos Humanos. Universidad Complutense de Madrid, 406. 
Nino, E. (2010). Efecto o intención: cual deberia ser el requisito en los casos de discriminacion. En M. d. Humanos, Igualdad y No discriminación. El reto de la diversidad (pág. 220). Quito: V\&M Gráficas.

Pietro, L. (marzo de 1994). Repositorio de la Universidad de Alicante. Recuperado de https://rua.ua.es/dspace/bitstream/10045/10632/1/doxa15-16_18.pdf

Ruiz, R. (s.f.). Tesis Doctorales en Red. Recuperado de http://www.tdx.cat/bitstream/handle/10803/10750/RuizCarbonell.pdf 